\title{
Magnetism of perovskite cobaltites with Kramers rare-earth ions
}

\author{
Z. Jirák, ${ }^{1, a)}$ J. Hejtmánek, ${ }^{1}$ K. Knížek, ${ }^{1}$ P. Novák, ${ }^{1}$ E. Šantavá, ${ }^{1}$ and H. Fujishiro ${ }^{2}$ \\ ${ }^{1}$ Institute of Physics ASCR, Cukrovarnická 10, 16200 Prague 6, Czech Republic \\ ${ }^{2}$ Faculty of Engineering, Iwate University, Morioka 020-8551, Japan
}

(Presented 6 November 2013; received 19 September 2013; accepted 28 October 2013; published online 27 January 2014)

\begin{abstract}
The band-gap insulators $R E \mathrm{CoO}_{3}\left(R E=\mathrm{Nd}^{3+}, \mathrm{Sm}^{3+}\right.$, and $\left.\mathrm{Dy}^{3+}\right)$ with $\mathrm{Co}^{3+}$ ions stabilized in the non-magnetic low-spin state have been investigated by specific heat measurements. The experiments evidence an antiferromagnetic ordering of the rare earths with Néel temperature of $T_{N}=1.25,1.50$, and $3.60 \mathrm{~K}$ for $\mathrm{NdCoO}_{3}, \mathrm{SmCoO}_{3}$, and $\mathrm{DyCoO}_{3}$, respectively. With increasing external field, the lambda peak in specific heat, indicative of the transition, shifts to lower temperatures and vanishes for field of about $3 \mathrm{~T}$. Starting from this point, a broader Schottky peak is formed, centered in $1 \mathrm{~K}$ range, and its position is moved to higher temperatures proportionally to applied field. The origin of the peak is in Zeeman splitting of the ground Kramers doublet, and the gradual shift with field defines effective $g$-factors for the rare-earth pseudospins in studied compounds. The results obtained are confronted with the calculations of crystal field splitting of the rare-earth multiplets. (C) 2014 AIP Publishing LLC. [http://dx.doi.org/10.1063/1.4862946]
\end{abstract}

The rare-earth cobaltites $R E \mathrm{CoO}_{3}$ possess a distorted perovskite structure of the orthorhombic space group $\mathrm{P}$ bnm, characterized by a tilt of $\mathrm{CoO}_{6}$ octahedra and location of rare-earth ions in sites of low point symmetry $C_{s}$ (the mirror plane $m$ ). Since the octahedrally coordinated $\mathrm{Co}^{3+}$ ions are stabilized in a non-magnetic low-spin state, the magnetic properties depend primarily on the existence of $R E^{3+}$ moments and their (dipole-dipole or superexchange) interactions. It is essential that the free-ion multiplets of rare earths are split by crystal field effects, resulting in Kramers doublets for ions with odd number of $4 f$ electrons and singlets for ions with the even number. In the case of a non-magnetic singlet state, the low-temperature magnetic property is van Vleck susceptibility arising due to mixing in external field of the ground singlet with higher-lying levels. The well-known example is $\operatorname{Pr}^{3+}\left(4 f^{2}\right)$ showing linear paraprocess up to very high fields, see e.g., Ref. 1. Exceptions of this rule are $\mathrm{Tb}^{3+}$ $\left(4 f^{8}\right)$ and $\mathrm{Ho}^{3+}\left(4 f^{10}\right)$ with accidental degeneracy of two lowest singlets, forming a magnetic pseudodoublet.

The present work is focused to the cobaltite systems with $\mathrm{Nd}^{3+}\left(4 f^{3}\right), \mathrm{Sm}^{3+}\left(4 f^{5}\right)$, and $\mathrm{Dy}^{3+}\left(4 f^{9}\right)$ ions that exhibit Kramers degeneracy and display, therefore, finite moments (the $J^{\prime}=1 / 2$ pseudospins) in the ground state. The study combines the low-temperature heat capacity experiments and extensive calculations of the rare-earth levels and their magnetic characteristics.

The determination of the $\mathrm{Nd}^{3+}, \mathrm{Sm}^{3+}$, and $\mathrm{Dy}^{3+}$ electronic levels was performed in two steps. Since the cobaltites (unlike the rare-earths aluminates) are non-transparent and no optical $f-f$ transitions are available, the crystal field parameters had to be first calculated. For this task, a novel method based on the first-principles electronic structure and Wannier projection ${ }^{2,3}$ was applied. The parameters, derived for the crystallographic data of real P bnm structures of the

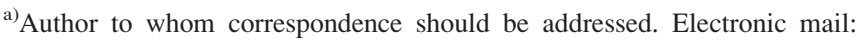
jirak@fzu.cz.
}

respective $R E \mathrm{CoO}_{3},{ }^{4-6}$ have been recently published in the Appendix of Ref. 7.

In the next step, the local Hamiltonian operating on the $4 f$ states in a determinantal basis of one-electron wavefunctions was constructed, including both the crystal field and Zeeman interaction terms. The eigenvalue problem was solved using "Lanthanide" program. ${ }^{8}$ The calculations were done for different orientations and strengths of the applied field, so that not only the crystal field split levels but also their magnetic characteristics were determined.

The crystal field splittings of free-ion multiplets of $\mathrm{Nd}^{3+}$ $\left({ }^{4} I_{9 / 2}\right), \mathrm{Sm}^{3+}\left({ }^{6} H_{5 / 2}\right)$, and $\mathrm{Dy}^{3+}\left({ }^{6} H_{15 / 2}\right)$ result in five, three, and eight Kramers doublets, respectively. Their calculated energy schemes are available in Ref. 7. In addition, the anisotropic $g_{J^{\prime}}$-factors and Van Vleck susceptibilities relative to the doublets are tabulated for the orientation of applied fields along the orthorhombic a, b, c axes and in the diagonal direction [110]. Following the $C_{s}$ symmetry of rare-earth sites, the principal axes of respective tensors are $z \| c$ and $x, y \perp c$, with $x$ inclined to $\pm \alpha$ from the $a$ axis (here \pm refers to two nonequivalent sites in $\mathrm{P}$ bnm structure). For the ground doublet of $\mathrm{Nd}^{3+}$, the principal components of $g_{J^{\prime}}$-tensor are $g_{x}=2.818, g_{y}=1.228, g_{z}=3.015$, and the angle of local $x$-axis makes an angle $\alpha_{g}= \pm 62.3^{\circ}$ with the orthorhombic $a$ axis. The next doublet is situated at an excitation energy $\Delta=13.2 \mathrm{meV}$, which means that it makes no contribution to rare-earth moments in the investigated temperature range of $T<30 \mathrm{~K}$. The data obtained for the ground doublet of $\mathrm{Sm}^{3+}$ are $g_{x}=0.703, g_{y}=0.588$, and $g_{z}=0.322$, the orientation of the $x$-axis under the angle $\alpha_{g}= \pm 33.2^{\circ}$ and the first excitation energy $\Delta=29.6 \mathrm{meV}$. The situation for $\mathrm{Dy}^{3+}$ is specific by Ising character of the ground doublet, $g_{x}=19.44, g_{y} \sim 0$, and $g_{z} \sim 0$, where the orientation of the Ising axis is under the angle $\alpha_{g}= \pm 64.0^{\circ}$ with the orthorhombic $a$ axis. The first excitation energy is calculated to $\Delta=29.8 \mathrm{meV}$.

In the experimental part, ceramic samples $\mathrm{NdCoO}_{3}$, $\mathrm{SmCoO}_{3}$, and $\mathrm{DyCoO}_{3}$ were prepared using a solid-state 

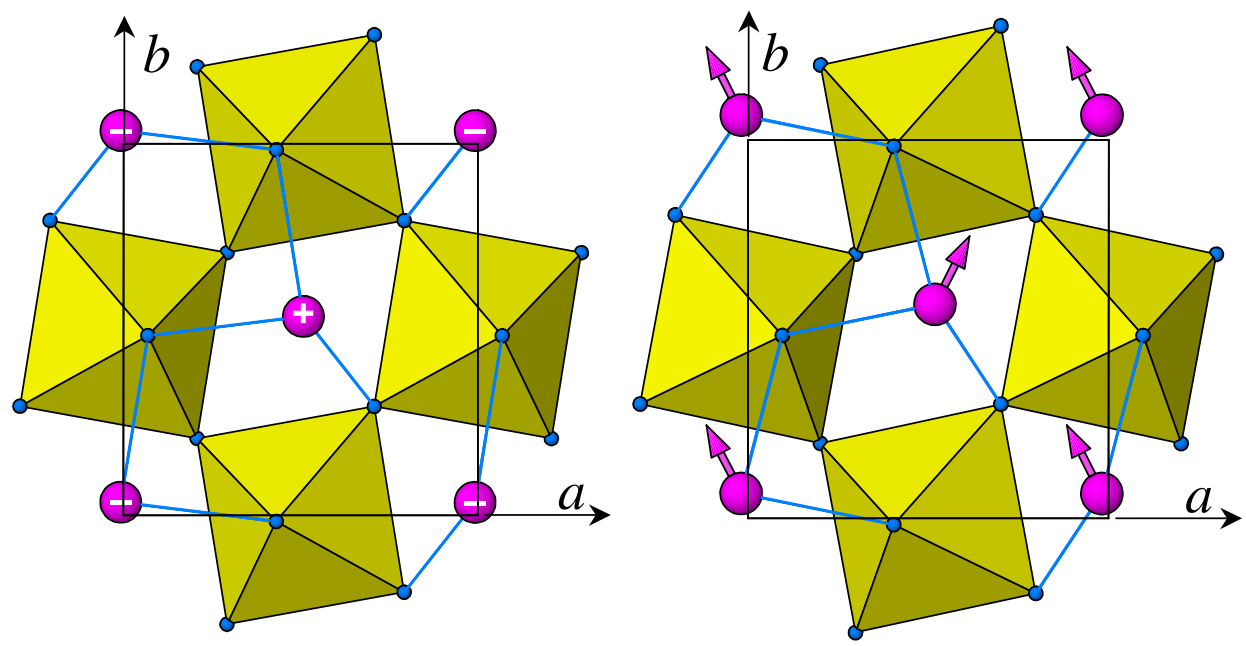

FIG. 1. The AFM arrangement of $C_{z}$ type in $\mathrm{NdCoO}_{3}$ below $T_{N}=1.25 \mathrm{~K}$ [Ref. 9] (left) and $G_{x} A_{y}$ in $\mathrm{DyCoO}_{3}$ below $T_{N}=3.60 \mathrm{~K}$ [Ref. 7] (right). The scheme shows the octahedral tilts and rare-earth moments located at the mirror plane at $c=1 / 4$ level, while those at $c=3 / 4$ are oppositely oriented. The blue lines mark the shortest $R E-\mathrm{O}$ bonds.

reaction. Raw powders of $\mathrm{Nd}_{2} \mathrm{O}_{3}, \mathrm{Sm}_{2} \mathrm{O}_{3}, \mathrm{Dy}_{2} \mathrm{O}_{3}$, and $\mathrm{Co}_{3} \mathrm{O}_{4}$ were weighted with proper molar ratios and ground using an agate mortar and pestle for $1 \mathrm{~h}$. Mixed powders were calcinated at $1000{ }^{\circ} \mathrm{C}$ for $24 \mathrm{~h}$ in air. They were pulverized and ground. Then, they were pressed into pellets of $20 \mathrm{~mm}$ diameter and $4 \mathrm{~mm}$ thickness. Pellets were sintered at $1300^{\circ} \mathrm{C}$ for $24 \mathrm{~h}$ under air. The measured densities of each sample were greater than $90 \%$ of the ideal density. Powder X-ray diffraction patterns were taken for each sample using $\mathrm{CuK}_{\alpha}$ radiation; the samples were confirmed to have a single phase orthoperovskite $\mathrm{P}$ bnm structure. The lattice parameters and volume per f.u. actually obtained are $a=5.344 \AA, b=5.335 \AA, c=7.548 \AA$, $V / Z=53.79 \AA^{3}$ for $\mathrm{NdCoO}_{3}, \quad a=5.283 \AA, \quad b=5.350 \AA$, $c=7.496 \AA, V / Z=52.97 \AA^{3}$ for $\mathrm{SmCoO}_{3}$, and $a=5.170 \AA$, $b=5.410 \AA, c=7.397 \AA, V / Z=51.73 \AA^{3}$ for $\mathrm{DyCoO}_{3}$. The present values are in an agreement with the literature data for the same compounds.

The specific heat was measured by Physical Properties Measuring System (Quantum Design) using the two- $\tau$ model. The experiments at very low temperatures (down to $0.4 \mathrm{~K}$ ) were done using the ${ }^{3} \mathrm{He}$ option. The data were taken on sample cooling at several runs, in zero field and selected fields up to $90 \mathrm{kOe}\left(140 \mathrm{kOe}\right.$ for $\left.\mathrm{SmCoO}_{3}\right)$. The data for $\mathrm{NdCoO}_{3}$ in Fig. 2 show a sharp $\lambda$-peak at $T_{N}=1.25 \mathrm{~K}$, which evidences an antiferromagnetic ordering found for this compound by neutron diffraction, actually the $C_{z}$-type arrangement in Bertaut's notation, ${ }^{9}$ see Fig. 1. With increasing external field, this $\lambda$-peak shifts to lower temperatures and vanishes for field of about $30 \mathrm{kOe}$. Starting from this point, a broader Schottky peak is formed, centered in $1 \mathrm{~K}$ range, and its position is moved to higher temperatures proportionally to applied field. Origin of this peak is in Zeeman splitting of the ground state doublet, and the gradual shift with field defines an effective $g$-factor for the $\mathrm{Nd}^{3+}$ pseudospins. A detailed analysis reveals a broadening with respect to ideal Schottky form, which is in coherence with the pseudoaxial anisotropy of $\mathrm{Nd}^{3+}$ moments, which can be expressed, using the calculated principal components $g_{x}=2.818, g_{y}=1.228$, and $g_{z}=3.015$ for the ground doublet, by a ratio $2 g_{y} /\left(g_{x}+g_{z}\right)=0.42$. Indeed, the best experimental fit in the lower panel of Fig. 2 is achieved for the ratio $g_{\|} / g_{\perp} \sim 0.35$ and 0.54 for $\mathrm{H}=50$ and $90 \mathrm{kOe}$, respectively. The average value $g=2.29$ determined from the field shift of Schottky peak for our polycrystalline sample (see the inset) is also in a reasonable agreement with the theoretical value $\langle g\rangle=2.44$.

Similar $\lambda$-peak is observed for $\mathrm{SmCoO}_{3}$ at $T_{N}=1.50 \mathrm{~K}$ (Fig. 3). The detailed type of AFM arrangement is, however, uncertain since no neutron diffraction data are available for this compound. This is due to extreme absorption of natural samarium for neutrons which can be, nonetheless, overcome by isotopic substitution. The AFM ordering is much more robust in external fields than for $\mathrm{NdCoO}_{3}$, so that the $\lambda$-peak though decreasing and shifting to lower temperatures is observed up to $90 \mathrm{kOe}$, where formation of Schottky peak just starts. In still higher fields, only a slow upward shift of Schottky maximum is observed. The reason is in a weak Zeeman energy of $\mathrm{Sm}^{3+}$. This follows from near canceling

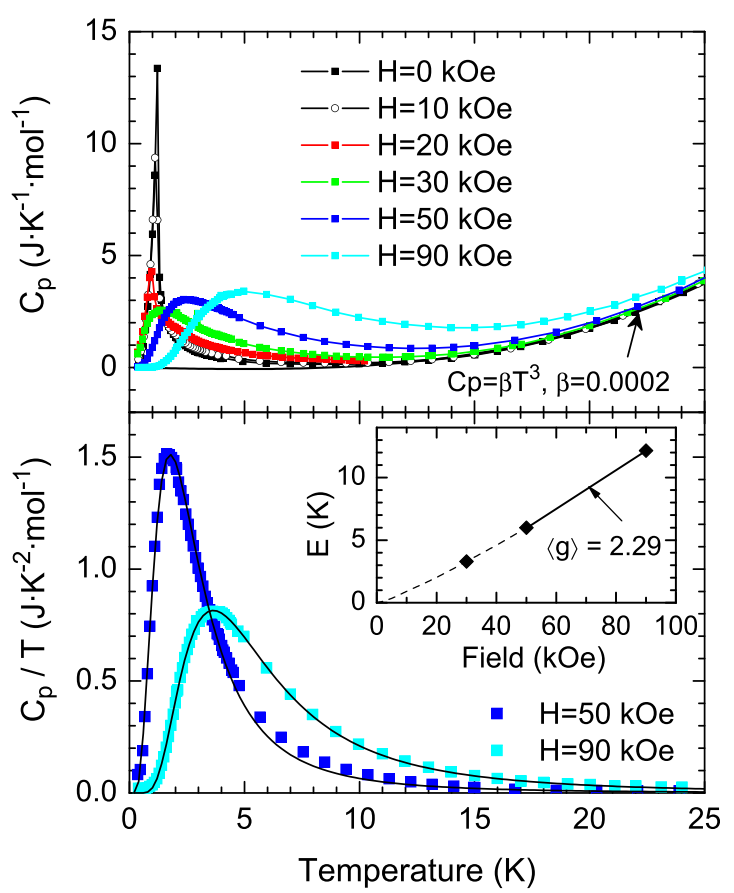

FIG. 2. The specific heat of $\mathrm{NdCoO}_{3}$ in dependence on applied field. The lower panel shows the least-squares fit of Schottky peak form, supposing the axial symmetry of the $g$-factor. The average Zeeman splitting energy is presented in the inset. 


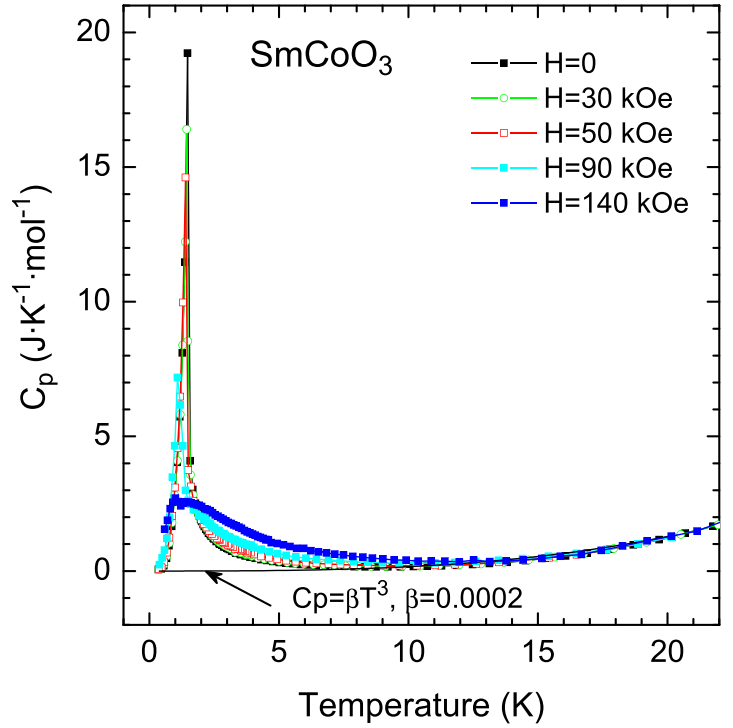

FIG. 3. The specific heat of $\mathrm{SmCoO}_{3}$ in dependence on applied field.

of the spin and orbital moments in ${ }^{6} H_{5 / 2}$ multiplet $(L=5$, $\left.S=5 / 2, J=L-S=5 / 2, g_{L}=2 / 7\right)$, which is manifested among others by low $g_{J^{\prime}}$-factor of the pseudospins in ground Kramers doublet $\left(g_{x}=0.703, g_{y}=0.588\right.$, and $\left.g_{z}=0.322\right)$.

For $\mathrm{DyCoO}_{3}$, the AFM ordering is of a non-collinear $G_{x} A_{y}$-type, see the right picture in Fig. 1. Such canted arrangement is a consequence of Ising character of the $g_{J^{\prime}}$-tensor of $\mathrm{Dy}^{3+}$ ions and zigzag orientation of their easy axes in the P bnm structure. ${ }^{7,10}$ The zero-field heat capacity data in (Fig. 4) show $T_{N}=3.60 \mathrm{~K}$, but the AFM ordering quickly vanishes in external field, which should be related to a strong Zeeman energy associated with $g_{\|}=19.44$ of the Dy ${ }^{3+}$ pseudospins. Upon the collapse of the sharp $\lambda$-peak, a very diffusive Schottky-like peak arises and broadens with increasing field. Finally, it acquires the form of a notably large linear term that adds to the common cubic term of lattice heat. This behavior of $\mathrm{DyCoO}_{3}$ is a natural consequence of Ising moments in polycrystalline materials. Namely, when external field is oriented under random angles $\theta$ to local Ising axes, the Zeeman splitting varies as $\cos \theta$ and, instead of two sharp levels, the polycrystal as a whole exhibits a continuous spectrum of excitations with constant density of states, spreading from $\Delta E=0$ to $\Delta E_{\max .}{ }^{11}$ The constant density of

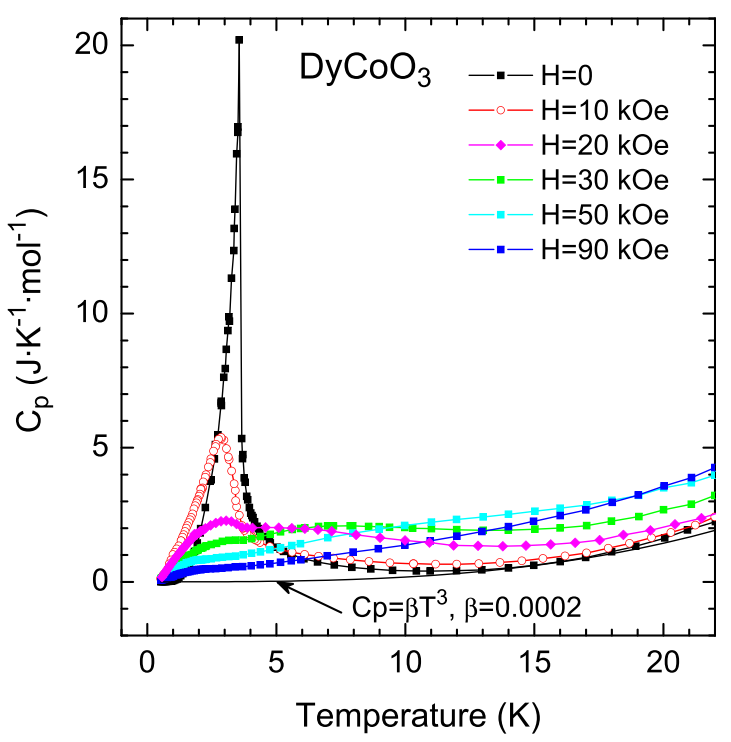

FIG. 4. The specific heat of $\mathrm{DyCoO}_{3}$ in dependence on applied field.

states then gives the strictly linear low-temperature term of specific heat.

This work was supported by Project No. P204/11/0713 of the Grant Agency of the Czech Republic.

${ }^{1}$ R. M. Thomas, V. Skumryev, J. M. D. Coey, and S. Wirth, J. Appl. Phys. 85, 5384 (1999).

${ }^{2}$ P. Novák, K. Knížek, and J. Kuneš, Phys. Rev. B 87, 205139 (2013).

${ }^{3}$ P. Novák, K. Knížek, M. Maryško, Z. Jirák, and J. Kuneš, J. Phys.: Condens. Matter 25, 4460001 (2013).

${ }^{4}$ K. Knížek, J. Hejtmánek, Z. Jirák, P. Tomeš, P. Henry, and G. André, Phys. Rev. B 79, 134103 (2009).

${ }^{5}$ J. Pérez-Cacho, J. Blasco, J. García, and R. Sanchez, J. Solid State Chem. 150, 145 (2000).

${ }^{6}$ J. A. Alonso, M. J. Martínez Lope, C. de la Calle, and V. Pomjakushin, J. Mater. Chem. 16, 1555 (2006).

${ }^{7}$ K. Knížek, P. Novák, Z. Jirák, and C. R. dela Cruz, Solid State Sciences 28, 26 (2014).

${ }^{8}$ S. Edvardsson and D. Aberg, Comput. Phys. Commun. 133, 396 (2001).

${ }^{9}$ I. Plaza, E. Palacios, J. Bartolomé, S. Rosenkranz, C. Ritter, and A. Furrer, Physica B 234-236, 632 (1997).

${ }^{10}$ A. Kappatsch, A. Quezel-Ambrunaz, and J. Sivardiére, J. Phys. France 31, 369 (1970).

${ }^{11}$ J. M. D. Coey and S. von Molnar, J. Phys. Lett. 39, L327 (1978). 